\title{
Two New Distribution Families Constructed by a Modified Rank Transmutation
}

\author{
Mehmet Yilmaz* and Sibel Acik Kemaloglu \\ (Communicated by Kamile Sanli KULA)
}

\begin{abstract}
In this study, two new distribution families are derived by modifying the rank transmutation. The base distribution of one of these two families is $F^{2}$, and the other is $F$. The modification on the rank transmutation allowed to extend the range of the transmutation parameter from $[-1,1]$ to $[-1,2]$. At this point, the generated distributions gain more flexibility. Considering exponential distribution as the base distribution, the importance and flexibility of the proposed families are illustrated by two applications. Since the structure of the transmuted family with base distribution is simpler, the statistical and reliability properties of this family are examined. Furthermore, some orderings based on the survival and hazard rate revealed.
\end{abstract}

Keywords: quadratic rank transmutation; rank transmutation; modified rank transmutation; transmuted distribution. AMS Subject Classification (2010): Primary: 60E05 Secondary: 62E10.

${ }^{*}$ Corresponding author

\section{Introduction}

Let's consider the quadratic rank transmutation map (QRTM) proposed by Shaw and Buckley (2007), [13]. Then the mapping is given by

$$
u \rightarrow u+\lambda u(1-u)
$$

where $u \in[0,1]$ and $\lambda \in[-1,1]$. Using this transformation many distributions have been derived and still continue to be derived. Beside this, there are also some studies on the modifications of the QRTM. Some of the pioneering works on proposing modified QRTM can be given as follows: Abd El Hady (2014), [1] proposed a new Weibull distribution by using exponentiated QRTM. Alizadeh et al. (2015), [2] generated a new distribution family by considering exponentiated distribution as a baseline distribution. Merovci (2013), [10] studied a new distribution by taking a baseline distribution as exponentiated exponential distribution. Eltehiwy and Ashour (2013), [5] introduced transmuted exponentiated modified Weibull distribution, and Ashour and Eltehiwy (2013), [3] introduced transmuted exponentiated Lomax distribution. These last three studies can be seen as a special case of Alizadeh et al. (2015), [2]. Mansour et al. (2015), [8] introduced new transmutation map by adding extra two parameters to get more flexible distribution. Then, Mansour and Mohamed (2015), [9] introduced a new Lindley distribution by using this new transmutation map approach. Das and Barman (2015), [4] introduced a kind of generalization of QRTM by considering sum of k- dimensional vector of transmutation parameters. There are two similar studies that respectively are the generalized transmuted G family by Nofal et al. (2017), [11] and generalized transmuted Weibull distribution by Nofal and El Gebaly (2017), [12].

Received : 01-07-2017, Accepted : 04-01-2018 
In this study, a new modified transmutation has been proposed in the light of the idea behind QRTM. Here, the range of the transmutation parameter is extended from the interval $[-1,1]$ to the interval $[-1,2]$. At this point, the distribution has become more flexible. In order to demonstrate the usefulness of the gained flexibility of the distribution family, an illustrative example is given on the real data set in the application section.

\section{Construction of the Family}

Let's start with the multiplication of the transmutation defined by the mapping in eq. (1.1) with $u$. Then, we have the following transmormation named as $\pi(u)$

$$
\pi(u)=u[u+\lambda u(1-u)]=u^{2}+\lambda u^{2}(1-u)=(1+\lambda) u^{2}-\lambda u^{3}
$$

The transformation obtained in this way is well defined and many distributions can be derived. However, here the base distribution is the distribution of the maximum of two independent copies generated from uniform distribution, namely the baseline is $u^{2}$. Now let's check the range of the transmutation parameter $\lambda$. Currently, the range of transmutation parameter still lies between -1 and 1. Now let's see how we can extend the range of $\lambda$. Taking $1+\lambda=3 \delta$, where $\delta \in[0,1]$. Then, $\lambda \in[-1,2]$. In this case, let's try to rewrite eq. (2.1). Then we have

$$
\pi(u)=3 \delta u^{2}+(1-3 \delta) u^{3}=\delta\left(3 u^{2}-2 u^{3}\right)+(1-\delta) u^{3}
$$

As can be seen now, the inequality $3 u^{2}-2 u^{3} \leq u^{3}$ is provided. For this reason, $\pi(u)$ can be expressed as the convex combination of these two statements. Furthermore, $u^{3}$ represents the distribution function of the maximum of the three independent copies from uniform distribution. Similarly, $3 u^{2}-2 u^{3}$ represents the distribution function of the median of these copies. According to these explanations, there is no doubt that the range of the transmutation parameter is the closed interval $[-1,2]$. The distribution function of any continuous random variable $X$ is denoted by $F(x)$. The distribution function obtained by the above extended rank transmutation is denoted by $G(x)$. By setting $u=F(x)$ in eq. (2.1) we have a new transmuted distribution defined by

$$
G(x)=(1+\lambda) F^{2}(x)-\lambda F^{3}(x)
$$

where $\lambda \in[-1,2]$. We call in shortly the transmuted distribution obtained by this way as G-T-D. At this time, many distributions can be obtained by this transformation, but the base distribution is $F^{2}$ at $\lambda=0$. That is, the transformed distribution is not $F$ but the distribution function of the maximum of two independent copies, such as $X_{1}$ and $X_{2}$. In order to make the base distribution as $F$, a square root transformation can be applied to $\pi(u)$. So, taking $\pi^{*}(u)=\sqrt{\pi(u)}$ we have another transmutation given by

$$
G^{*}(x)=F(x) \sqrt{(1+\lambda)-\lambda F(x)}=F(x) \sqrt{1+\lambda \bar{F}(x)}
$$

where, $\bar{F}=1-F$ denotes the survival function. Taking into account on eq. (2.2) which represents a convex combination, $g(x)=\frac{d G(x)}{d x} \geq 0$ holds for all $x$. Thus, $g(x)$ represents the probability density function of Gtransmuted random variable $X$ for $\lambda \in[-1,2]$. Now, with the following theorem, we check whether $g^{*}(x)$ can be represented as a pdf for $\lambda \in[-1,2]$, just like $g(x)$.

Theorem 2.1. $G^{*}(x)$ is a nondecresing function of $x$, for $\lambda \in[-1,2]$ and the function $g^{*}(x)$ is the probability density function of the random variable from the distribution $G^{*}$.

Proof. The first derivative of $G^{*}(x)$ with respect to $x$ is written as

$$
\frac{d G^{*}(x)}{d x}=f(x) \sqrt{1+\lambda \bar{F}(x)}-\frac{\lambda f(x) F(x)}{2 \sqrt{1+\lambda \bar{F}(x)}}=\frac{f(x)}{2 \sqrt{1+\lambda \bar{F}(x)}}[2-\lambda+3 \lambda \bar{F}(x)]
$$

Hence, the sign of this derivative is a non-negative for $\lambda \geq 0$. The statement in square brackets can be written as $[2(1+\lambda \bar{F}(x))-\lambda F(x)]$. Then, the sign of this derivative is a non-negative also for $\lambda \in[-1,0)$. Thus, the first part of the proof is completed. In this case, $g^{*}(x) \geq 0$ is satisfied if the derivative expression is denoted as $g^{*}(x)$. Furthermore, $\int_{-\infty}^{\infty} g^{*}(x) d x=1$ must be provided by $g^{*}(x)$. This integral can be expressed as:

$$
\int_{-\infty}^{\infty} g^{*}(x) d x=\lim _{a \rightarrow \infty}\left(G^{*}(a)-G^{*}(-a)\right)=\lim _{a \rightarrow \infty} F(a) \sqrt{1+\lambda \bar{F}(a)}-\lim _{a \rightarrow-\infty} F(a) \sqrt{1+\lambda \bar{F}(a)}
$$


Since $F$ denotes a distribution function and $\bar{F}$ denotes respective survival function, both $\lim _{a \rightarrow \infty} F(a)=\lim _{a \rightarrow-\infty} \bar{F}(a)=1$ and $\lim _{a \rightarrow-\infty} F(a)=\lim _{a \rightarrow \infty} \bar{F}(a)=0$ are satisfied. Then, the integral above is evaluated as $\lim _{a \rightarrow \infty} F(a) \sqrt{1+\lambda \bar{F}(a)}-$ $\lim _{a \rightarrow-\infty} F(a) \sqrt{1+\lambda \bar{F}(a)}=1-0=1$. Thus, $g^{*}(x)$ denotes also a probability density function.

Now, any distribution generated by eq. (2.4) is named as $G^{*}-T-D$. As a result, two distribution families, such as $G$ and $G^{*}$ are defined. Due to the complex nature of the $G^{*}$ family, it is difficult to examine its mathematical characteristics. However, thanks to advances in technology, the increased software quality and computer processor speeds, the usefulness of the $G^{*}$ family will be demonstrated on real data sets. In the following sections of the study, until the application section, only the G-T-D family will be studied.

\section{Statistical Properties of G-Transmuted Family}

In this section, the alternative forms of the cumulative distribution functions (cdf) and probability density function (pdf) are examined, then the raw moments and moment generating function are given in the light of these definitions.

\subsection{The cdf of G-transmuted family}

Let $X_{r: n}$ be a $r t h$ order statistic obtained from a sample of size $n$ from the population whose distribution is $F(x)$. Let $F_{r: n}$ and $f_{r: n}$ denote cdf and pdf of $X_{r: n}$, respectively. Then eq. (2.3) can be rearranged as follows:

$$
\begin{aligned}
G(x) & =(1+\lambda) F_{2: 2}(x)-\lambda F_{3: 3}(x) \\
& =F^{2}(x)[1+\lambda \bar{F}(x)] \\
& =\left(\frac{1+\lambda}{3}\right) F_{2: 3}(x)+\left(\frac{2-\lambda}{3}\right) F_{3: 3}(x)
\end{aligned}
$$

We call the random variable $T$ as $G-T$ random variable and its distribution, $G$ called as $G-T-D$. Following theorem is about stochastic ordering relations between $G$ and $F$.

Theorem 3.1. Let $T$ and $X$ be continuous random variables with respective cdf $G$ and $F$. Then the following relations hold.

i. $T_{\lambda^{\prime}} \leq{ }_{s t} T_{\lambda}$ for $\lambda \leq \lambda^{\prime}$

ii. $X \leq_{s t} T$ for $\lambda \in[-1,1]$, and $T \leq_{s t} X$ for $\lambda \in(1,2]$

iii. $X \leq_{s t} X_{2: 2} \leq_{s t} T$ for $\lambda \in[-1,0], X \leq_{s t} T \leq_{s t} X_{2: 2}$ for $\lambda \in(0,1]$, and $T \leq_{s t} X \leq_{s t} X_{2: 2}$ for $\lambda \in(1,2]$

iv. $X_{2: 3} \leq_{s t} T \leq_{s t} X_{3: 3}$ for $\lambda \in[-1,2]$

Proof. i. Since $\frac{d G_{\lambda}(x)}{d \lambda}=F_{2: 2}(x)-F_{3: 3}(x)=F^{2}(x) \bar{F}(x) \geq 0 G_{\lambda}(x)$ is a nondecreasing function of $\lambda$. Hence, $G_{\lambda}(x) \leq G_{\lambda^{\prime}}(x)$ holds for $\lambda \leq \lambda^{\prime}$. This gives desired result.

ii. It follows from the inequalities for the ratio $\frac{G(x)}{F(x)}$ that are $F(x)[1+\lambda \bar{F}(x)] \leq 1$, when $\lambda \in[-1,1]$, and $\frac{G(x)}{F(x)} \geq 1$ when $\lambda \in(1,2]$.

iii. The mean lifetime of the parallel system constructed of two independent and identical parts, is always bigger than the mean lifetime of a single part. Accordingly, note that the ordering $X \leq_{s t} X_{2: 2}$ is always provided since $F^{2} \leq F$. Then, the relation $G \leq F^{2} \leq F$ holds for $\lambda \leq 0$. On the other hand, for $\lambda>0$ the inequality $F^{2} \leq G$ is provided. Taking into account (ii), for $\lambda \in(0,1]$ the relation $F^{2} \leq G \leq F$ is obtained and for $\lambda \in(1,2]$ the obtained relation is $F^{2} \leq F \leq G$.

iv. It is clear, since $G$ can be represented by a convex combination of $F_{2: 3}$ and $F_{3: 3}$. 
3.2 The pdf of G-transmuted family

According to eq. (3.1), the pdf of the G-T random variable is given by the following subsequent equalities:

$$
\begin{aligned}
g(x) & =(1+\lambda) f_{2: 2}(x)-\lambda f_{3: 3}(x) \\
& =f(x) F(x)[2+2 \lambda-3 \lambda F(x)] \\
& =f(x) F(x)[2-\lambda+3 \lambda \bar{F}(x)] \\
& =\left(\frac{1+\lambda}{3}\right) f_{2: 3}(x)+\left(\frac{2-\lambda}{3}\right) f_{3: 3}(x)
\end{aligned}
$$

3.3 The raw moments and moment generating function of G-transmuted family

From the eq. (2.2) and eq. (3.2), kth raw moment of $G-T$ random variable, $T$ is given by

$$
\begin{aligned}
E\left[T^{k}\right] & =(1+\lambda) E\left[X_{2: 2}^{k}\right]-\lambda E\left[X_{3: 3}^{k}\right] \\
& =\left(\frac{1+\lambda}{3}\right) E\left[X_{2: 3}^{k}\right]+\left(\frac{2-\lambda}{3}\right) E\left[X_{3: 3}^{k}\right]
\end{aligned}
$$

Respectively, moment generating function of $T$ is given by

$$
\begin{aligned}
M_{T}(v) & =(1+\lambda) M_{X_{2: 2}}(v)-\lambda M_{X_{3: 3}}(v) \\
& =\left(\frac{1+\lambda}{3}\right) M_{X_{2: 3}}(v)+\left(\frac{2-\lambda}{3}\right) M_{X_{3: 3}}(v)
\end{aligned}
$$

\section{Random Number Generation from G-Transmuted Family}

The number generation process will be done with convex combination notation given in eq. (2.2) and with the another form of G-T-D given by the eq. (3.1). At this point, it is possible to describe the G-T-D as a two-component mixture distribution, and then the random number generation from $G-T-D$ will be easier.

Step 1: Generate three random numbers from the baseline distribution, namely $X_{1}, X_{2}, X_{3} \sim F^{2}$

Step 2: Generate a random number $U$ from uniform distribution on $(0,1)$.

Step 3: If $\sqrt{U} \leq \frac{1+\lambda}{3}$, a random number $T$ from G-T-D is $X_{2: 3}$, or $\max \left\{\min \left\{X_{1}, X_{2}\right\}, \min \left\{X_{1}, X_{3}\right\}, \min \left\{X_{2}, X_{3}\right\}\right\}$, otherwise $T=X_{3: 3}=\max \left\{X_{1}, X_{2}, X_{3}\right\}$.

\section{Survival and Hazard Rate Functions of G-T Random Variable}

The survival function of a $G-T$ random variable is given by

$$
\begin{aligned}
\bar{G}(x) & =(1+\lambda) \bar{F}_{2: 2}(x)-\lambda \bar{F}_{3: 3}(x) \\
& =\bar{F}(x)\left[1+F(x)-\lambda F^{2}(x)\right] \\
& =\left(\frac{1+\lambda}{3}\right) \bar{F}_{2: 3}(x)+\left(\frac{2-\lambda}{3}\right) \bar{F}_{3: 3}(x)
\end{aligned}
$$

Note that, the survival function lies between the survival functions of $X_{2: 3}$ and $X_{3: 3}$. The other characteristic of interest of a random variable is the hazard rate function also known as failure rate function defined by $r(x)=$ $\frac{-d \log (\bar{G}(x))}{d x}$. The hazard rate function for a G-T random variable given by

$$
\begin{aligned}
r(x) & =r_{F}(x)\left[1-\frac{\bar{F}(x)[1-2 \lambda F(x)]}{1+F(x)-\lambda F^{2}(x)}\right] \\
& =r_{F}(x)\left[3-\frac{3+(1-2 \lambda) F(x)}{1+F(x)-\lambda F^{2}(x)}\right]
\end{aligned}
$$

The hazard rate function of the G-T random variable has some properties which are given by following theorem.

Theorem 5.1. Hazard rate function of the G-T random variable, i.e. $r(x)$ provides the following properties: 
(i) $r_{F_{3: 3}}(x) \leq r(x) \leq r_{F_{2: 3}}(x)$,

(ii) For $\lambda \leq \frac{1}{2}$, and $\lambda=2$, if $r_{F}(x)$ is constant or nondecreasing, $r(x)$ is non-decreasing,

(iii) $\lim _{x \rightarrow-\infty} r(x)=0$, and $\lim _{x \rightarrow \infty} r(x)=r_{F}(\infty)$,

(iv) $r(x) \leq r_{F}(x)$ holds for $\lambda \leq \frac{1}{2}$.

Proof. (i) By taking the first derivative of the second expression in square brackets of the eq. (5.2) with respect to the $\lambda$, and setting $F(x)=u$, we have

$$
\frac{d}{d \lambda}\left[\frac{3+(1-2 \lambda) u}{1+u-\lambda u^{2}}\right]=\frac{-u(1-u)(2+u)}{\left(1+u-\lambda u^{2}\right)^{2}} \leq 0
$$

Hence, $r_{\lambda}(x)$ nondecreases in $\lambda$. Namely, $r_{-1}(x)=r_{F_{3: 3}}(x) \leq r_{2}(x)=r_{F_{2: 3}}(x)$ holds.

(ii) By taking the first derivative of the same expression with respect to $u$, we have the following equation

$$
\frac{d}{d u}\left[3-\frac{3+(1-2 \lambda) u}{1+u-\lambda u^{2}}\right]=\frac{3(1-2 \lambda u)-(1-2 \lambda)\left(1+\lambda u^{2}\right)}{\left(1+u-\lambda u^{2}\right)^{2}}
$$

For the sake of brevity, the expression given by the numerator is denoted by $\rho(u)$. We now need to check the sign of $\rho(u)$ to determine monotonicity of this derivation. For $\lambda \geq-1$ the inequality $1-2 \lambda \leq 3$ is provided. Using this inequality, a lower bound for $\rho(u)$ is obtained as $\rho(u) \geq-3 \lambda u(2+u)$. Consequently, it can be immediately seen that the sign of this derivative is non-negative for $\lambda \leq 0$. On the other hand, the inequality $1-2 \lambda u \geq 1-2 \lambda$ is satisfied for $\lambda \geq 0$. Hereby, a lower bound for $\rho(u)$ can be obtained as $\rho(u) \geq(1-2 \lambda)\left(2-\lambda u^{2}\right)$. This lower bound is non-negative for $\lambda \in\left[0, \frac{1}{2}\right]$. Therefore, the first part of the proof is completed. For $\lambda=2, \rho(u)=6(1-u)^{2} \geq 0$ is satisfied, and hence the desired result is reached.

(iii) It is obvious.

(iv) It is clear from the first equality of eq. (5.2), so $\lambda \leq \frac{1}{2}$ implies that the inequality $1-2 \lambda F(x) \geq 0$ holds for all $x$.

\section{Application}

In this section the exponential distribution is only considered as the base distribution $F$ since applicability of exponential distribution is fairly wide. We study two real data sets to illustrate the usefulness of G-T-Exponential and $G^{*}-T$-Exponential distributions for modeling real data. We make comparison of the results with exponential and Transmuted exponential distributions. In order to compare canditate distributions, we will consider that Kolmogorov-Smirnov statistics (K-S), as the goodness-of-fit statistics, and Akaike Information Criterion (AIC), as the model selection criteria.

The goodness-of-fit statistics, K-S and the model selection criteria, AIC are described as follows:

- Goodness-of-fit statistics: Kolmogorov- Smirnov $K-S=\sup _{x}\left|G(x)-G_{n}(x)\right|$, where $G_{n}(x)$ is the empirical distribution function.

- Akaike Information Criterion: $A I C=-2 \log L(\Theta ;)+2 k$, where $k$ is the size of the parameter vector $\Theta$.

Data Set1. (100 bank customer data) The data set represents the waiting times (in minutes) before serving 100 bank customers which is given by $0.8,0.8,1.3,1.5,1.8,1.9,1.9,2.1,2.6,2.7,2.9,3.1,3.2,3.3,3.5,3.6,4.0,4.1,4.2,4.2$, 4.3, 4.3, 4.4, 4.4, 4.6, 4.7, 4.7, 4.8, 4.9, 4.9, 5.0, 5.3, 5.5, 5.7, 5.7, 6.1, 6.2, 6.2, 6.2, 6.3, 6.7, 6.9, 7.1, 7.1, 7.1, 7.1, 7.4, 7.6, 7.7, 8.0, 8.2, 8.6, 8.6, 8.6, 8.8, 8.8, 8.9, 8.9, 9.5, 9.6, 9.7, 9.8, 10.7, 10.9, 11.0, 11.0, 11.1, 11.2, 11.2, 11.5, 11.9, 12.4, 12.5, 12.9, 13.0, 13.1, 13.3, 13.6, 13.7, 13.9, 14.1, 15.4, 15.4, 17.3, 17.3, 18.1, 18.2, 18.4, 18.9, 19.0, 19.9, 20.6, 21.3, 21.4, 21.9, 23.0, 27.0, 31.6, 33.1, 38.5. This data set was modelled with Lindley distribution by Ghitany et al. (2008), [6].

We try to model this data set by using Exponential, Transmuted Exponential, G-T-Exponential and $G^{*}-T$ Exponential distributions. Table1 shows the ML estimates of the model parameters, Kolmogorov-Smirnov statistics, (K-S), and Akaike Information Criterion, (AIC). 
Table 1. Model statistics for bank customer data

\begin{tabular}{|l|l|l|l|}
\hline Model & ML estimates & K-S (p value) & AIC \\
\hline Exponential & $\hat{\theta}=0.1012$ & $0.1730(0.0044)$ & 660.0418 \\
\hline T-Exponential & $\hat{\theta}=0.1517 ; \hat{\lambda}=-1.0000$ & $0.0469(0.9730)$ & 638.5156 \\
\hline G-T-Exponential & $\hat{\theta}=0.1597 ; \hat{\lambda}=-0.2268$ & $0.0384(0.9973)$ & 638.3706 \\
\hline$G^{*}$-T-Exponential & $\hat{\theta}=0.1290 ; \hat{\lambda}=-1.0000$ & $0.0959(0.2974)$ & 643.9565 \\
\hline
\end{tabular}

According to Table 1, G-T-Exponential distribution fits better than the other considered models.

Data Set2. The data set contains the time intervals (in days) between coal mine accidents caused death of 10 or more men which is given by $378,96,59,108,54,275,498,228,217,19,156,36,124,61,188,217,78,49,271,120,329$, $47,15,50,1,233,113,17,131,208,275,330,129,31,120,13,28,32,1205,182,517,20,312,1630,215,203,189,22,23$, $644,255,1613,66,171,29,11,176,345,61,151,467,195,54,291,145,217,137,55,20,78,361,871,224,326,4,75,7$, $4,93,81,99,312,48,566,1312,369,364,18,15,59,286,326,354,123,390,348,338,37,1357,72,315,114,275,58$, 457, 72, 745, 336, 19. This data set was obtained by Maguire et al. (1952), [7]. Statistical values of G-T-Exponential distribution are not tabulated in Table 2 since $p$-value of K-S test statistics is $0.0014<0.05$.

Table 2. Model statistics for coal mine accidents data

\begin{tabular}{|l|l|l|l|}
\hline Model & ML estimates & K-S (p value) & AIC \\
\hline Exponential & $\hat{\theta}=238.0952$ & $0.0906(0.3129)$ & 1415.6 \\
\hline T-Exponential & $\hat{\theta}=357.1429 ; \hat{\lambda}=0.6722$ & $0.0627(0.7603)$ & 1412.2 \\
\hline$G^{*}$-T-Exponential & $\hat{\theta}=325.1817 ; \hat{\lambda}=1.4435$ & $0.0584(0.8295)$ & 1411.3 \\
\hline
\end{tabular}

According to K-S test results in Table 2, G*-T-Exponential distribution has the best fit within possible candidate distributions.

\section{Conclusion}

In this paper, we propose two families of the distributions named as $G-T$ and $G^{*}-T$ by modifying rank transmutation map. This modification led the range of transmutation parameter to extend from $[-1,1]$ to $[-1,2]$. Obviously this extension provides more flexibility to analyze real life data. We show that the proposed transform obtained by multiplying QRTM by $u$ is actually a convex combination of failure distributions of 3-out-of-1 and 3-out-of-2 systems. Then, the G-T-D family is proposed. Thus, G-T-D can be expressed as a mixture of the cdfs of the maximum and the middle of three independent copies from the base distribution. The other proposed family of distribution, $G^{*}$-T-D family, is only obtained by taking the square of the proposed transformation. Thanks to technological improvements, it can be useful in data modeling, but it is difficult to obtain mathematical or statistical properties theoretically.

Since the structure of the $G-T-D$ has a simpler form than $G^{*}-T-D$, we provide some mathematical and statistical properties for the G-T-D family. We derive expressions for the cdf, pdf, raw moments and generating functions of G-T-D. We also study some reliability characteristics like survival function and hazard rate function. We discuss stochastic and hazard rate ordering properties. We consider G-T-Exponential and $G^{*}$-T-Exponential distributions by taking the exponential distribution as the base distribution. The proposed distributions applied to a real data set provide better fits than exponential and transmuted exponential distributions. We hope that the proposed models will attract wider application in areas such as engineering, hydrology, medicine and economics, etc.

\section{References}

[1] Abd El Hady, N. E., Exponentiated Transmuted Weibull Distribution. International Journal of Mathematical, Computational, Statistical, Natural and Physical Engineering (2014), 8(6).

[2] Alizadeh, M., Merovci, F., and Hamedani, G. G., Generalized transmuted family of distributions: properties and applications. Hacettepe Journal of Mathematics and Statistics, forthcomig, (2015).

[3] Ashour, S. K., and Eltehiwy, M. A., Transmuted exponentiated Lomax distribution. Aust J Basic Appl Sci, 7(7), (2013), 658-667. 
[4] Das, K. K., and Barman, L., On some generalized transmuted distributions. Int. J. Sci. Eng. Res, 6, (2015), 1686-1691.

[5] Eltehiwy, M., and Ashour, S., Transmuted exponentiated modified Weibull distribution. International Journal of Basic and Applied Sciences, 2(3), (2013), 258-269.

[6] Ghitany, M. E., Atieh, B., and Nadarajah, S., Lindley distribution and its application. Mathematics and computers in simulation, 78(4), (2008), 493-506.

[7] Maguire, B. A., Pearson, E. S., and Wynn, A. H. A., The time intervals between industrial accidents. Biometrika, 39(1/2), (1952), 168-180.

[8] Mansour, M. M., Enayat, M. A., Hamed, S. M., and Mohamed, M. S., A new transmuted additive Weibull distribution based on a new method for adding a parameter to a family of distributions. Int. J. Appl. Math. Sci, $8,(2015), 31-51$.

[9] Mansour, M. M., and Mohamed, S. M., A new generalized of transmuted Lindley distribution. Appl. Math. Sci, 9, (2015), 2729-2748.

[10] Merovci, F., Transmuted exponentiated exponential distribution. Mathematical Sciences and Applications E-Notes, $1(2),(2013)$.

[11] Nofal, Z. M., Afify, A. Z., Yousof, H. M., and Cordeiro, G. M., The generalized transmuted-G family of distributions. Communications in Statistics-Theory and Methods, 46(8), (2017), 4119-4136.

[12] Nofal, Z. M., and El Gebaly, Y. M., The Generalized Transmuted Weibull Distribution for Lifetime Data. Pakistan Journal of Statistics and Operation Research, 13(2), (2017), 355-378.

[13] Shaw, W.T and Buckley, I.R.C., The Alchemy of Probability Distributions: Beyond Gram-Charlier and CornishFisher Expansions, and Skew-Normal or Kurtotic-Normal Distributions. Research report, 2007.

\section{Affiliations}

MEHMET YiLMAZ

AdDRESS: Ankara University, Dept. of Statistics, Ankara-Turkey. E-MAIL: mehmetyilmaz@ankara.edu.tr

ORCID ID: $0000-0002-9762-6688$

Sibel ACiK Kemaloglu

ADDRESS: Ankara University, Dept. of Statistics, Ankara-Turkey.

E-MAIL: acik@ankara.edu.tr

ORCID ID: $0000-0003-0449-6966$ 